\title{
COMPRESSED SAMPLING INEQUALITIES \\ BY TCHAKALOFF'S THEOREM
}

\author{
MARCo Vianello
}

Abstract. We show that a discrete version of Tchakaloff's theorem on the existence of positive algebraic cubature formulas, entails that the information required for multivariate polynomial approximation can be suitably compressed.

Mathematics subject classification (2010): 41A10, 65D32.

Keywords and phrases: Tchakaloff's theorem, multivariate polynomial inequalities, compressed sampling.

\section{REFERENCES}

[1] C. Bayer And J. Teichmann, The proof of Tchakaloff's theorem, Proc. Amer. Math. Soc. 134 (2006), 3035-3040.

[2] T. Bloom, L. Bos, J.-P. CAlvi And N. Levenberg, Polynomial interpolation and approximation in $\mathbb{C}^{d}$, Ann. Polon. Math. 106 (2012), 53-81.

[3] L. Bos, J.-P. Calvi, N. Levenberg, A. Sommariva and M. Vianello, Geometric Weakly Admissible Meshes, Discrete Least Squares Approximations and Approximate Fekete Points, Math. Comp. 80 (2011), 1601-1621.

[4] L. Bos, S. De Marchi, A. Sommariva And M. Vianello, Weakly Admissible Meshes and Discrete Extremal Sets, Numer. Math. Theory Methods Appl. 4 (2011), 1-12.

[5] L. BOS AND M. VIANELLO, Low cardinality admissible meshes on quadrangles, triangles and disks, Math. Inequal. Appl. 15 (2012), 229-235.

[6] J.-P. CALVI AND N. LEVENBERG, Uniform approximation by discrete least squares polynomials, J. Approx. Theory 152 (2008), 82-100.

[7] S. De Marchi, F. Piazzon, A. Sommariva And M. Vianello, Wam: Matlab package for polynomial fitting and interpolation on Weakly Admissible Meshes (software available online at: http://www.math.unipd.it/ marcov/CAAsoft.html).

[8] M. Gentile, A. Sommariva And M. Vianello, Polynomial interpolation and cubature over polygons, J. Comput. Appl. Math. 235 (2011), 5232-5239.

[9] A. Kroó, On optimal polynomial meshes, J. Approx. Theory 163 (2011), 1107-1124.

[10] C. L. LAWSON AND R. J. HANSON, Solving least squares problems, Classics in Applied Mathematics 15, SIAM, Philadelphia, 1995.

[11] F. Piazzon And M. Vianello, Small perturbations of polynomial meshes, Appl. Anal. 92 (2013), 1063-1073.

[12] F. Piazzon and M. Vianello, Constructing optimal polynomial meshes on planar starlike domains, Dolomites Res. Notes Approx. DRNA 7 (2014), 22-25.

[13] W. PleśniaK, Multivariate Jackson Inequality, J. Comput. Appl. Math. 233 (2009), 815-820.

[14] M. Putinar, A note on Tchakaloff's theorem, Proc. Amer. Math. Soc. 125 (1997), 2409-2414.

[15] A. Sommariva AND M. Vianello, Compression of multivariate discrete measures and applications, Numer. Funct. Anal. Optim. 36 (2015), 1198-1223.

[16] V. TCHAKalofF, Formules de cubature mécaniques à coefficients nonnégatifs, Bull. Sci. Math. 81 (1957), 123-134. 